\title{
MONITORING OF HEMOSTASIS BY ROTATIONAL THROMBELASTOMETRY DURING NORMAL PREGNANCY AND POSTPARTUM
}

\author{
Duraj L ${ }^{1}$, Stasko $\mathrm{J}^{1}$, Hasko $\mathrm{M}^{2}$, Chudy $\mathrm{P}^{3}$, Sokol $\mathrm{J}^{1}$, Danko $\mathrm{J}^{2}$, Kubisz $\mathrm{P}^{1}$ \\ ${ }^{1}$ National Center of Thrombosis and Haemostasis, Clinic of Haematology and Transfusiology, Jessenius Faculty \\ of Medicine, Comenius University and University Hospital in Martin, Martin, Slovakia \\ ${ }^{2}$ Clinic of Gynecology and Obstetrics, Jessenius Faculty of Medicine, Comenius University and University \\ Hospital in Martin, Martin, Slovakia \\ ${ }^{3}$ Department of Haematology and Blood Transfusion, KNsP Cadca, Cadca, Slovakia
}

\begin{abstract}
Background: Rotational thrombelastometry (ROTEM) is a real-time clotting test that provides insight into clotting factors, the fibrinolytic system and platelet function. We obtained the longitudinal values on ROTEM in normal pregnancy and in puerperium.

Material and Methods: After ethics committee approval and subject informed consent, citrated blood was sampled from healthy pregnants four times during pregnancy and one time postpartum. As controls we used nonpregnant women undergoing voluntary blood donation. Extem and Intem tests and basic coagulation test were carried out.

Results: We included 112 women in our study, 55 non-pregnant women (controls) and 57 healthy pregnants with 5 samplings. The values of maximum clot firmness (MCF - in EXTEM and INTEM) were significantly higher up to 34 th - 36th week of pregnancy than those in non-pregnant subjects. MCF in 6 th -7 th week after delivery was significantly higher in both tests. Clotting time (CT) in pregnant women was significantly shorter (EXTEM) compared to non-pregnant subjects. We also found a very strong correlation between MCF and platelet count in all gestational weeks.

Conclusions: Rotation thromboelastometry clearly demonstrates the hypercoagulability in pregnancy and can reflect the higher risk of venous thromboembolism in both pregnancy and puerperium. Strong correlation between MCF and platelet count can suggest role of platelets in hypercoagulability in pregnant women. This study provides a better knowledge about physiological changes in ROTEM measurement during pregnancy and postpartum.
\end{abstract}

Key words: rotation thromboelastometry, thromboelastography, global hemostasis test, maternal hemostatic change.

\section{INTRODUCTION}

Pregnancy is a hypercoagulable state with increased thrombotic risk throughout gestation and the postpartum (Papa, 2009). The main causes of hypercoagulation during pregnancy are a slowdown of blood flow, a reduction of protein $\mathrm{S}$ activity, an increase in prothrombin activity and higher concentration of some plasma coagulation factors (fibrinogen, factors VII, VIII and von Willebrand factor). There also exist some physiological compensation mechanisms such a pregnancy hemodilution and an increase in tissue factor-pathway inhibitor (TFPI) activity. Even with the existence of these compesation mechanisms hypercoagulation arises in physiological pregnancy (1).

Despite this hypercoagulability, postpartum hemorrhage remains the leading cause of maternal death and is often complicated by coagulation problems. Delays in obstetric management and in the identification and correction of coagulation problems are crucial prognostic factors for severe postpartum hemorrhage (2). Unfortunately, conventional laboratory clotting techniques do not provide results rapidly and can not fully identify subjects with an increased thromboembolic risk. (3).

Address for correspondence:

Prof. Jan Stasko, MD, PhD, Clinic of Haematology and Transfusiology, Comenius University in Bratislava, Jessenius Faculty of Medicine and University Hospital in Martin, Kollarova Str. 2, 03601 Martin, Slovakia

E-mail: Jan.Stasko@jfmed.uniba.sk 
Rotational thrombelastometry (ROTEM) is a real-time clotting test. As a blood clot is formed between the cup and the pin, resistance against the oscillation is measured using computerised system. Results are given in a graphic presentation that provides insight into clotting factors, the fibrinolytic system and platelet function (4).

We hypothesize that coagulation changes significantly during pregnancy and after delivery. Previously studies by thromboelastometry/ thrombelastography in pregnancy have been an observational design, using non-pregnant women as controls $(1,2,5,6)$ (Othman 2010, Armstrong 2011, Huissoud 2009, Polak 2011). One study in healthy pregnancies used sample of the same women 6 week postpartum as controls (4). We performed longitudinal study with 4 samplings during pregnancy and 1 post-partum measurement and as a control we used young healthy women - blood donors.

Knowledge of the standard ROTEM values (cut-off) during pregnancy is necessary for diagnostic requirements of global coagulation and requisite in future studies of complicated pregnancies. This study provides a better overview of the changes in hemostasis during normal pregnancy, which can help in the future for diagnostics or tailored therapy of complicated pregnancies with hemostasis disorders.

\section{MATERIALS AND METHODS}

\section{Study design}

The research group constited of 57 pregnant women (average age 30.6, range 19-44) with repeated sampling during pregnancy in time T1 (10th-12th week), time T2a (18th-20th week), time T2b (25th- 27th week), time T3 (34th-36th week) and after puerperium in time PP (6th-8th week). Only women with an uncomplicated obstetric history were enrolled. The control group was constituted of 55 non-pregnant women (average age 30.5, range $18-45$ ) undergoing voluntary blood donation.

\section{Exclusion criteria}

The exlusion criteria for pregnant women were :

1) age below 18 years or above 45 years;

2) medical history of hemostasis defects and/or thromboembolic disease;

3) obesity (BMI > 30);

4) anticoagulation and/or antiplatelet treatment during pregnancy;

5) have undergone abortion or pregnancy loss in the last 6 months;

6) women who did not consent to participate in the study;

Before being admitted into study, all of the women underwent an informative interview with a physician and expressed their written consent to participate in the study. The Ethics Committee of the Jessenius Faculty of Medicine granted consent with performed study.

\section{Sample collection}

Platelet count, hemoglobin concentration and hematocrit were assessed with the cell counter (Beckman Coulter DxH 800) in venous blood collected in $3 \mathrm{ml}$ tubes containing EDTA. Blood samples for ROTEM analysis and conventional coagulation assays were collected in $2.7 \mathrm{ml}$ tubes containing sodium citrate $(0.109 \mathrm{M})$ and were drawn between $8 \mathrm{am}$ and $9 \mathrm{am}$. Samples were maintained at $37^{\circ} \mathrm{C}$ before and during procedure and ROTEM tests were performed within 30 minutes of taking the sample. Prothrombin time (PT) and activated partial thromboplastin time (aPTT) were assessed within 1 hour of sample collection, according to standard procedures and using the automated coagulation analyzer (Sysmex CA1500). 


\section{Thrombelastometry}

We used the modified rotation thrombelastogram analyser (ROTEM®; Pentapharm Ltd, Munich, Germany) in our research laboratory of hemostasis and thrombosis. System speeds up the analysis and allows a specific exploration of the related pathways of coagulation (i.e. intrinsic-extrinsic pathways). The new ROTEM system also provides an intuitive graphical interface and automatic pipetting system to facilitate use by clinicians. Depending on the parameters measured, ROTEM results are available as early as 15 minutes up to 1 hour. Sample of citrated blood (300L) were recalcified with star-TEM ${ }^{\circledR}$ reagent $\left(20 \mu \mathrm{L} \mathrm{CaCl}_{2}\right.$ $0.2 \mathrm{M}$ ) and activated by ex-TEM ${ }^{\circledR}$ reagent (recombinant tissue factor + phospholipids) for monitoring extrinsic pathway or activated by in-TEM ${ }^{\circledR}$ reagent (ellagic acid + phospholipids) for monitoring intrinsic pathway.

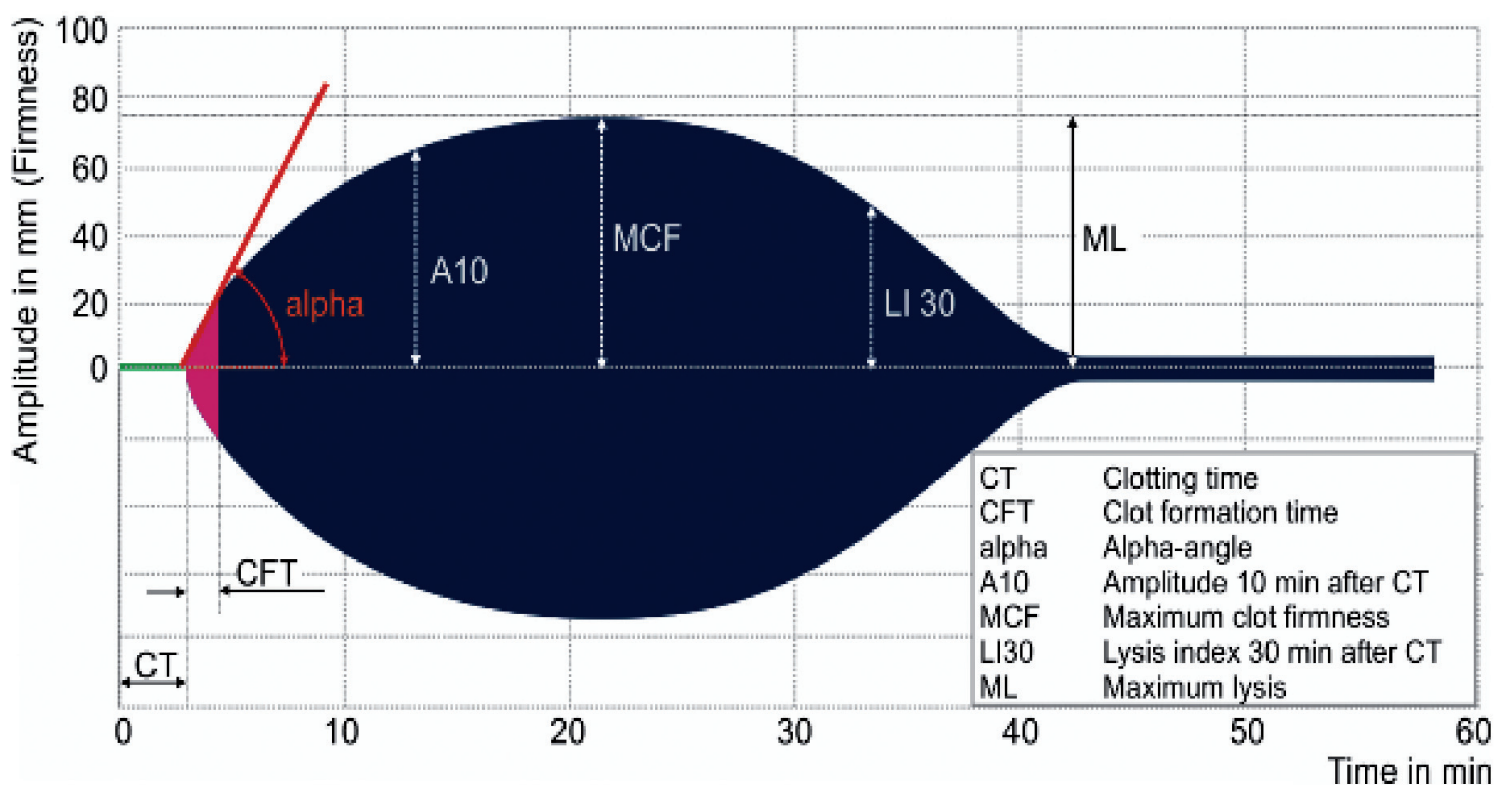

Fig. 1: Graphic representation of ROTEM parameters (7)

The ROTEM generates a reaction curve in real time based on numerical data calculated from the shape of the TEM-gram (Figure 1). The following variables were recorded from two channels, performing EXTEM and INTEM tests simultaneously. EXTEM test mildly activates hemostasis via the physiological activator tissue factor and is a screening test for the extrinsic hemostasis system. The result is influenced by extrinsic coagulation factors, platelets and fibrinogen. On the contrary, INTEM test mildly activates the contact phase of hemostasis by ellagic acid and monitors the intrinsic hemostasis system. The result is influenced by intrinsic coagulation factors, platelets, fibrinogen and heparin.

We measured clotting time (CT), maximum clot firmness (MCF) and clot lysis index at 30 min. (CLI 30). The method and classification of ROTEM parameters and clinical applications have been described in Table 1. 
Table 1 Classification of RO TEM parameters

\begin{tabular}{|l|l|l|l|}
\hline Curve parameter & Abbreviation & Definition & Clinical application \\
\hline Clotting time (s) & CT & $\begin{array}{l}\text { Time from start of test until the start of clot } \\
\text { formation }\end{array}$ & $\begin{array}{l}\text { Assesses activation of coagulation } \\
\text { primarily clotting factors }\end{array}$ \\
\hline Clot formation time (s) & CFT & $\begin{array}{l}\text { Time from beginning of clot formation to an } \\
\text { amplitude of } 20 \mathrm{~mm}\end{array}$ & Assesses rate of clot develoment \\
\hline Alpha angle & $\alpha$ & $\begin{array}{l}\text { The angle of a tangent to the curve at } 2 \mathrm{~mm} \\
\text { amplitude }\end{array}$ & $\begin{array}{l}\text { Assesses both rate and strength } \\
\text { of clot formation }\end{array}$ \\
\hline Maximum clot firmness (mm) & MCF & Maximal amplitude of the curve & $\begin{array}{l}\text { Assesses contribution of } \\
\text { fibrinogen and platelets to the clot }\end{array}$ \\
\hline Amplitude at set times (mm) & A5, A10 & Amplitude of the curve at 5, 10 min. & Assesses of rate of clot formation \\
\hline
\end{tabular}

\section{Statistical analysis}

We report results as medians and interquartile ranges. Statistical significance of the differences of values between patients and controls was calculated by the Mann-Whitney $\mathrm{U}$ test. The differences were considered statistically significant only for p-values less than 0.05. To evaluate the changes in ROTEM values during pregnancy, we used a Wilcoxon nonparametric test to compare the results for five time intervals (T1, T2a, T2b, T3, PP); p-values $<0.05$ were considered to be significant. The correlation between ROTEM data and results of standard coagulation tests was assessed by Spearman s rank correlation analysis. A pvalues $<0,05$ were considered to be significant. All statistical tests were carried out using SPSS 16.0® (SPSS Inc., Chicago, IL, USA).

\section{RESULTS}

We included 112 women in our study, 57 pregnant and 55 non-pregnant women (controls) according to the inclusion and exlusion criteria. Finally 50 pregnants of 57 were tested four times during pregnancy : time T1 $(n=57)$, time T2a $(n=53)$, time T2b $(n=52)$, time T3 $(n=50)$ and once after delivery : time PP $(n=50)$. The reasons for exclusion were inadequate blood sampling, developed pre-eclampsia, fetal loss or preterm delivery. We collected overall 317 measurements of each tests: blood count, routine coagulation tests and thrombelastometry tests (measurements of EXTEM test and INTEM test).

Hemoglobin concentration (HGB), hematocrit (HTC) and platelet counts (PLT) gradually decreased during pregnancy, but these values reverted in time PP (6 weeks after delivery) to the initial values in time T1 (Table 2). By contrast PT (11.6 vs. 11.5) and aPTT (30.0 vs. 27.4) did not change significantly during pregnancy. 
Table 2 Subject blood count and coagulation tests

\begin{tabular}{|c|c|c|c|c|c|}
\hline Groups & HGB & HTC & PLT & PT & aPTT \\
\hline Controls $(n=55)$ & $135[129-141]$ & $0.41[0.39-0.42]$ & $214[186-254]$ & $12.1[11.8-12.5]$ & $32.2[30.5-34.6]$ \\
\hline Group T1 $(n=57)$ & $130[126-134]$ & $0.38[0.37-0.39]$ & $236[203-261]$ & $11.6[11.4-12.0]$ & $30.0[28.5-31.1]$ \\
\hline Group T2a $(n=53)$ & $121[115-124]$ & $0.36[0.34-0.37]$ & $232[202-253]$ & $11.4[11.1-11.6]$ & $29.2[28.3-30.3]$ \\
\hline Group T2b $(n=52)$ & $115[108-119]$ & $0.34[0.33-0.36]$ & $229[191-256]$ & $11.4[11.0-11.7]$ & $28.0[27.0-29.4]$ \\
\hline Group T3 $(n=50)$ & $114[108-118]$ & $0.34[0.33-0.35]$ & $213[196-243]$ & $11.5[11.3-11.8]$ & $27.4[27.0-28.8]$ \\
\hline Group PP (n=50) & $128[121-133]$ & $0.38[0.37-0.39]$ & $254[219-280]$ & $11.6[11.1-11.8]$ & $29.8[28.7-31.5]$ \\
\hline
\end{tabular}

Note : Values are medians [interquartile range]; HGB $=$ Hemoglobin, HTC $=$ Hematocrit, PLT $=$ Platelets, PT $=$ Prothrombin time, aPTT $=$ Activated partial thromboplastin time.

The results obtained for EXTEM and intrinsic pathway INTEM are depicked in Table 3 for each group. CT in EXTEM test significantly decreased during all gestational ages and we observed the significant decrease after lying-in, too. Also CT in INTEM test significantly decreased during pregnancy, but not in all gestational ages (shortest values were between 25 th -27 th gestation week). By contrast, the values of CT in INTEM test reverted to normal values in group PP.

Table 3 RO TEM results of CT and MCF in Extem and Intem tests

\begin{tabular}{|c|c|c|c|c|}
\hline Groups & $\begin{array}{c}\text { EXTEM } \\
\text { CT }(s)\end{array}$ & $\begin{array}{c}\text { INTEM } \\
\text { CT }(s)\end{array}$ & $\begin{array}{c}\text { EXTEM } \\
\text { MCF }(\mathrm{mm})\end{array}$ & $\begin{array}{c}\text { INTEM } \\
\text { MCF }(\mathrm{mm})\end{array}$ \\
\hline Controls $(n=55)$ & $53[49-58]$ & $171[159-187]$ & $63[61-66]$ & $61[59-64]$ \\
\hline Group T1 $(n=57)$ & $56^{\star}[52-60]$ & $171[155-192]$ & $67^{\star \star \star}[65-69]$ & $64^{\star \star}[62-66]$ \\
\hline Group T2a $(n=53)$ & $52^{\star \star}[46-58]$ & $169[149-185]$ & $68^{\star \star \star}[66-70]$ & $66^{\star \star \star}[64-68]$ \\
\hline Group T2b $(n=52)$ & $45^{\star \star \star}[44-48]$ & $152^{\star \star}[142-160]$ & $69^{\star \star \star}[66-70]$ & $67^{\star \star \star}[65-69]$ \\
\hline Group T3 $(n=50)$ & $42^{\star \star \star}[39-45]$ & $156^{\star}[142-180]$ & $7^{\star \star \star \star}[69-73]$ & $71^{\star \star \star \star}[68-72]$ \\
\hline Group PP $(n=50)$ & $47^{\star \star \star}[44-49]$ & $169[157-188]$ & $66^{\star \star \star}[64-69]$ & $65^{\star \star}[62-67]$ \\
\hline
\end{tabular}

Note : Values are medians [interquartile range]; ns, $\mathrm{p}>0.05 ;{ }^{*} \mathrm{p}<0.05 ;{ }^{* *} \mathrm{p}<0.01 ;{ }^{* * *} \mathrm{p}<0.001$; $\mathrm{CT}=$ Clotting time, $\mathrm{MCF}=$ Maximum clot firmness. 
The most significant findings were found in MCF. The values of MCF were significantly increased in both INTEM and EXTEM tests (Table 3) with maximal peaks in the group closest to delivery (T3: 34th - 36th gestation week). We observed also significantly increased values of MCF in group PP compared to controls in each of both tests.

We assessed correlation between standard coagulation test results and clotting time of thrombelastometry. Significant correlations were observed between the CT in EXTEM test and PT results as well as between the CT in INTEM test and aPTT results (Table 4). There was a strong correlation between platelet count and MCF. The MCF significantly correlated in both tests with platelet count and the correlation coeficient was low almost in all gestational groups (Table 5).

Table 4 Correlation between RE TEM data and coagulation tests

\begin{tabular}{|c|c|c|c|c|}
\hline Groups & \multicolumn{2}{|c|}{ Correlation EXTEM CT : PT } & \multicolumn{2}{c|}{ Correlation CT : APTT } \\
\hline Controls $(n=55)$ & $r=0.286$ & $p<0.05$ & $r=0.573$ & $p<0.0001$ \\
\hline Group T1 $(n=57)$ & $r=0.428$ & $p<0.005$ & $r=0.266$ & $p=0.266$ \\
\hline Group T2a $(n=53)$ & $r=0.062$ & $p=0.062$ & $r=0.199$ & $p=0.199$ \\
\hline Group T2b $(n=52)$ & $r=0.046$ & $p=0.757$ & $r=0.788$ & $p=0.788$ \\
\hline Group T3 $(n=50)$ & $r=0.222$ & $p=0.147$ & $r=0.602$ & $p=0.602$ \\
\hline Group PP $(n=50)$ & $r=0.412$ & $p<0.005$ & $r=0.487$ & $p=0.487$ \\
\hline
\end{tabular}

Note : Coefficient of correlation "r" between standard coagulation test and ROTEM results for clotting time (CT) in Extem and Intem tests, Spearman's rank correlation analysis; aPPT: activated partial thromboplastin time; PT: prothrombin time.

Table 5 Correlation between RE TEM data and platelet count

\begin{tabular}{|c|c|c|c|c|}
\hline Groups & \multicolumn{2}{|l|}{ Correlation EXTEM MCF : PLT } & \multicolumn{2}{l|}{ Correlation INTEM MCF : PLT } \\
\hline Controls $(n=55)$ & $r=0.162$ & $p=0.246$ & $r=0.180$ & $p=0.198$ \\
\hline Group T1 $(n=57)$ & $r=0.401$ & $p<0.005$ & $r=0.510$ & $p<0.0001$ \\
\hline Group T2a ( $=53)$ & $r=0.488$ & $p<0.01$ & $r=0.576$ & $p<0.0001$ \\
\hline Group T2b $(n=52)$ & $r=0.500$ & $p<0.0001$ & $r=0.545$ & $p<0.0001$ \\
\hline Group T3 $(n=50)$ & $r=0.402$ & $p<0.01$ & $r=0.278$ & $p=0.074$ \\
\hline
\end{tabular}

Note: Coefficient of correlation " $r$ " between platelet count and ROTEM results for maximum clot firmness (MCF) in Extem and Intem tests, Spearman's rank correlation analysis, PLT = platelet count. 


\section{DISCUSSION}

The changes in coagulation observed in pregnancy and puerperium using thrombelastometry are consistent with a hypercoagulable state as similarly reported in previous studies $(1,2,5,8)$. Although the majority of our values were within the ranges set for non-pregnant healthy subjects, the coagulation was found to be increased dependently with gestational age. Similarly as van Rheenen-Flach et al. (4) we found the most significant increase in the MCF using both tests (INTEM and EXTEM), but highly significant changes we confirmed also in CT measured by EXTEM during all gestational periods. Using INTEM we found the significant decrease of CT in group T2b (25th- 27th gestational week) and T3 (34th-36th gestational week). Our findings of the significantly increased MCF (maximal peaks of MCF in 34th - 36th gestational week) are in concordance with the cross-sectional data reported by Huissoud et al. (2) as well as with prospective longitudinal data published by van Rheenen-Flach et al. (4). The MCF values of our pregnants were significantly higher than those in non-pregnant subjects (9). On the contrary to Huissoud et al. (2) who didn t observe changes of CT in any trimester but similarly as van Rheenen-Flach et al. (4) we found the significant decrease of CT with minimal values in 25th-36th weeks of pregnancy (EXTEM and INTEM).

By contrast to others the MCF in 6th - 7th week after delivery was significantly increased in our study using both tests (EXTEM and INTEM) and the CT was significantly decreased (shortered) (EXTEM) compared to non-pregnant subjects. These findings can support the idea that normalisation of hemostasis in puerperium is not completely achieved even up to 6 th -7 th week after labour. This results are opposite to data reported previously by Saha (10).

The increased amplitudes of MCF observed in our study can be associated with stronger viscoelastic forces in the clot reflecting the gestational age-dependent procoagulant changes in pregnancy. These results are tightly consistent with the development of hypercoagulation and can reflect the higher risk of venous thromboembolism in pregnancy. The clot strenght measured by ROTEM in FIBTEM test as MCF increases in a fibrinogen concentrationdependent manner (11). However, we also found a very strong correlation between MCF and platelet count (EXTEM and INTEM) in all gestational weeks in pregnancy (absent in nonpregnant subjects) which can suggest the important role of platelets in pathophysiology of hypercoagulability in pregnant women (12).

Despite described procoagulant changes in ROTEM results it is worthwile to mention that none of women included in the study developped any thromboembolic event.

\section{CONCLUSIONS}

This study provides more information about the physiological changes in ROTEM measurements during the different trimesters of pregnancy and provides reference ranges in healthy pregnant women. These data may serve as assistance for future studies and interventions, especially in cases where the clotting system is activated (e.g. preeclampsia) or challenged (e.g. hemorrhage).

\section{REFERENCES}

1. Polak F, Kolnikova I, Lips M, Parizek A, Blaha J, Stritesky M. New recommendations for thromboelastography reference ranges for pregnant women. Thromb Res 2011; 128: 14-17.

2. Huissoud C, Carrabin B, Benchaib M, Fontaine O, Levrat A, Massignon D, Touzet S, Rudigoz R, Berland M. Coagulation assessment by rotation thrombelastometry in normal pregnancy. Thromb Haemost 2009; $101: 755$ - 761.

3. Papa ML. Thromboelastometry, a Possible Tool to Identify Women at Risk of Pregnancy Loss. C TOATHERTJ 2009; 2:54-60. 
4. van Rheenen-Flach LE, Zweegman S, Boersma F, Lenglet JE, Twisk JWR, Bolte AC. A prospective longitudinal study on rotation thromboelastometry in women with uncomplicated pregnancies and postpartum. ANZJOG 2013; 53: 32-36.

5. Armstrong S, Fernando R, Ashpole K. Assessment of coagulation in the obstetric population using ROTEM ${ }^{\circledR}$ thromboelastometry. Int J Obstet Anesth 2011; 20: 293-298.

6. Othman M, Falcon B, Kadir R. Global hemostasis in pregnancy: are we using thromboelastography to its full potential? Semin Thromb Hemost 2010; 36: 738-746.

7. ROTEM - The bleeding management system. [cit. 2013-03-05] Access at webpage: http://www.rotem.de/site/index.php

8. Sharma SK, Philip J, Wiley J. Thromboelastographic changes in healthy parturients and postpartum women. Anesth Analg 1997; 85: 94.

9. Duraj L, Staško J, Kubisz P, Haško M, Chudý P, Sokol J, Šimonová R, Lisá L. Monitoring of hemostasis by thrombelastometry in normal pregnancy. Thromb Res 2012; 130: S143.

10. Saha P, Stott D, Atalla R. Haemostatic changes in the puerperium '6 weeks postpartum' (HIP Study) implication for maternal thromboembolism. BJOG 2009; 116: 1602-1612.

11. Solomon C, Rahe-Meyer N, Schőchl H, Ranucci M, Görlinger K. Effect of haematocrit on fibrin-based clot firmness in the FIBTEM test. Blood Transfus 2013; 11: 412-418.

12. Staško J, Duraj L, Haško M, Kubisz P, Danko J, Biringer K, Sokol J, Šimonová R, Lisá L. Is rotation thrombelastometry a useful method for monitoring of hemostasis in normal pregnancy and puerperium? ISTH 2013 ; c. $4.65-5$.

\section{Acknowledgements:}

The study was supported by projects „Center of Excellence for Perinatological Research I“ (CEPV I, ITMS 26220120016), which was cofinanced from EU sources, APVV 0222-11 and by Grant of Comenius University UK/319/2012.

Received: June, 10, 2015

Accepted: July, 11, 2015 